\title{
The Skeleton of a Reduced Word and a Correspondence of Edelman and Greene
}

\author{
Stefan Felsner \\ Freie Universität Berlin \\ Fachbereich Mathematik und Informatik \\ Takustr. 9 \\ 14195 Berlin, Germany \\ felsner@inf.fu-berlin.de
}

Submitted: July 31, 2000; Accepted: December 29, 2000

\begin{abstract}
Stanley conjectured that the number of maximal chains in the weak Bruhat order of $S_{n}$, or equivalently the number of reduced decompositions of the reverse of the identity permutation $w_{0}=n, n-1, n-2, \ldots, 2,1$, equals the number of standard Young tableaux of staircase shape $s=\{n-1, n-2, \ldots, 1\}$. Originating from this conjecture remarkable connections between standard Young tableaux and reduced words have been discovered. Stanley proved his conjecture algebraically, later Edelman and Greene found a bijective proof. We provide an extension of the Edelman and Greene bijection to a larger class of words. This extension is similar to the extension of the Robinson-Schensted correspondence to two line arrays. Our proof is inspired by Viennot's planarized proof of the Robinson-Schensted correspondence. As it is the case with the classical correspondence the planarized proofs have their own beauty and simplicity.
\end{abstract}

Key Words. Chains in the weak Bruhat order, reduced decompositions, Young tableaux, bijective proof, planarization.

Mathematics Subject Classifications (2000). 05E10, 05A15, 20F55.

\section{Introduction}

Stanley conjectured in [14] that the number of maximal chains in the weak Bruhat order of $S_{n}$, or equivalently the number of reduced decompositions of the reverse of the identity permutation $w_{0}=n, n-1, n-2, \ldots, 2,1$, equals the number $f_{s}$ of standard Young tableaux

An extended abstract of this paper has appered in the proceedings of FPSAC'00 (see [3]) 
of staircase shape $s=\{n-1, n-2, \ldots, 1\}$. Evaluating $f_{s}$ with the hook-formula yields

$$
\left|\operatorname{Red}\left(w_{0}\right)\right|=\frac{\left(\begin{array}{l}
n \\
2
\end{array}\right) !}{(2 n-3) \cdot(2 n-5)^{2} \cdot(2 n-7)^{3} \cdot \ldots \cdot 5^{n-3} \cdot 3^{n-2}} .
$$

Originating from this conjecture some remarkable connections between standard Young tableaux and reduced words have been discovered and explained. Stanley [15] proved the original conjecture algebraically. Edelman and Greene [2] found a bijective proof. Further proofs are given by Lascoux and Schützenberger [13] and Haiman [9].

The basic correspondence has been generalized in different directions. Based on conjectures of Stanley [15] a related correspondence between shifted standard tableaux and reduced decompositions of the longest element in the hyperoctahedral group, i.e., the Weyl group of type $B_{n}$, was established by Kraskiewicz [12] and Haiman [9]. In recent work Fomin and Kirillov [5] found an amazing generalization of Stanley's formula which includes a formula of Macdonald as a second special case.

The main purpose of this paper is to give a planarized construction and proof for the bijection of Edelman and Greene between reduced words and certain pairs of Young tableaux. The construction is similar in spirit to the planarization of the RobinsonSchensted correspondence of Viennot $[17,18]$. In particular we introduce a skeleton for reduced words. We agree with Viennot's statement [18, page 412]: "Unfortunately the simplicity of the combinatorial constructions, together with the magic of this very beautiful correspondence, cannot be written down in a paper as easily as it can be described in an oral communication with a friend or using superposition of pictures with transparencies".

In the next section we give a rather broad introduction to the background of our construction. In Subsection 2.1 we indicate the relation between reduced words of permutations and partial arrangements. Subsection 2.2 is an exposition of the proof of the Robinson-Schensted correspondence using the geometric construction of skeletons as introduced by Viennot. In Subsection 2.3 we state the bijection of Edelman and Greene between reduced decompositions and certain pairs of Young tableaux. Along the lines of Viennot's proof we introduce the terminology required for our geometric version of this bijection. At the end of this subsection we state our main theorem which is a generalization of the Edelman and Greene bijection. The proof of the theorem is given in Section 3. We conclude in Section 4 by indicating a possible extension of the present work.

\section{Preliminaries}

In this section we introduce the set-up for the main bijection of this paper. We explain the connection between reduced decompositions and arrangements. After that Viennot's planarized version of the Robinson-Schensted correspondence is reviewed. Finally, we present the Edelman-Greene bijection. To prepare for the planarized proof we introduce switch diagrams and their skeleton. The section concludes with the statement of the planarized bijection. The proof of the theorem is given in the next section. 


\subsection{Reduced Words and Arrangements}

The weak Bruhat order of $S_{n}$, denoted $W B_{n}$ is the ordering of all permutations $\sigma$ of $[n]$ by inclusion of their inversion sets $\operatorname{Inv}(\sigma)=\left\{\left(\sigma_{i}, \sigma_{j}\right): i<j\right.$ and $\left.\sigma_{i}>\sigma_{j}\right\}$, i.e,

$$
\sigma \leq_{W B} \tau \Longleftrightarrow \operatorname{Inv}(\sigma) \subseteq \operatorname{Inv}(\tau) .
$$

The cover relation in $W B_{n}$ consists of the pairs $(\sigma, \tau)$ where $\tau$ is obtained from $\sigma$ by exchanging two adjacent elements which are in increasing order, i.e., $\sigma \leq_{W B} \tau$ and $\mid \operatorname{InV}(\sigma) \backslash$ $\operatorname{Inv}(\tau) \mid=1$. The unique minimal element of the weak Bruhat order is the identity permutation id $=1,2, \ldots, n$ and the unique maximal element is the reverse of the identity, $w_{0}=n, n-1, \ldots, 1$. The weak Bruhat order is a graded lattice with rank function $r(\sigma)=|\operatorname{Inv}(\sigma)|$. A maximal chain in $W B_{n}$ is a sequence of $\left(\begin{array}{l}n \\ 2\end{array}\right)+1$ permutations beginning with id and ending with $w_{0}$. Figure 1 shows the Hasse diagram of $W B_{4}$, this graph is also known as the 1-dimensional skeleton of the permutahedron. Maximal chains in $W B_{n}$ are known to have several interesting interpretations, below we describe two of these, another interpretation as reflection network is described by Knuth [11].

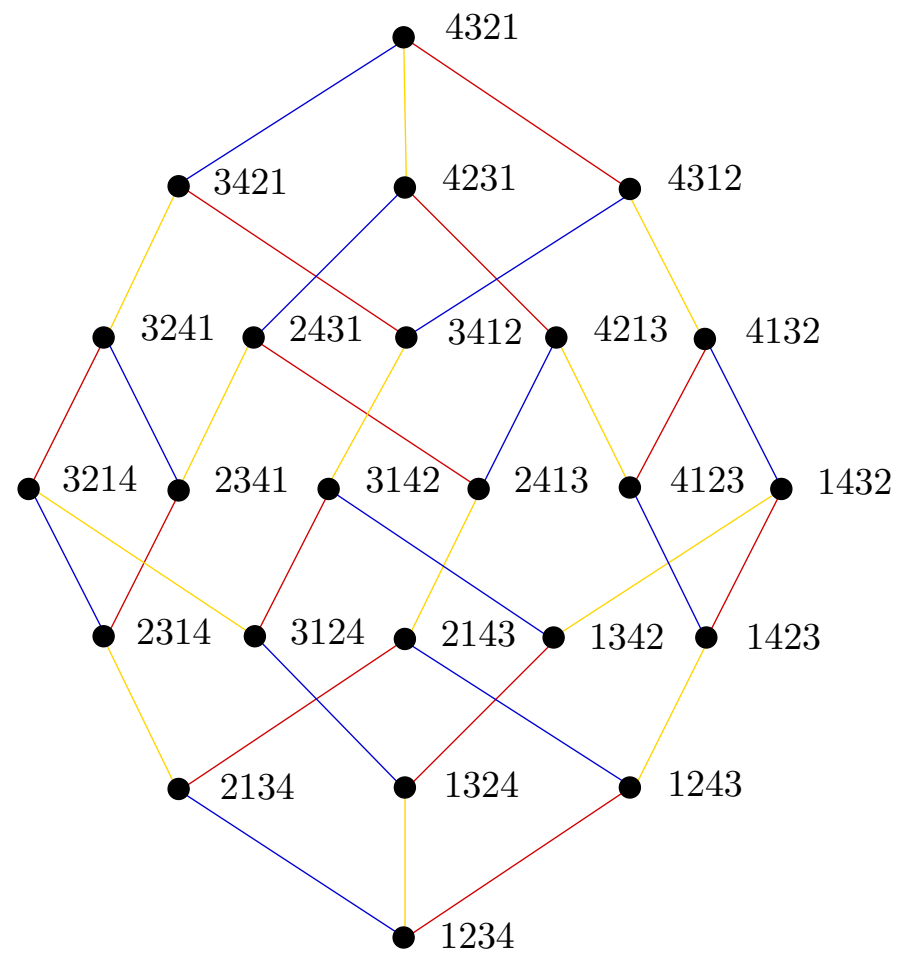

Figure 1: The diagram of the weak Bruhat order $W B_{4}$ of $S_{4}$.

Color the edges of the cover graph of $W B_{n}$ with the elements of $N=\{1, \ldots, n-1\}$ such that edge $(\sigma, \tau)$ is colored $i$ exactly if the two permutations $\sigma$ and $\tau$ differ by a transposition exchanging positions $i$ and $i+1$. Note that every permutation is incident to exactly one edge of every color. If we fix id as the start permutation we can associate 
to every word $\omega$ over the alphabet $N$ a unique walk in the cover graph of $W B_{n}$. With a word $\omega$ associate the permutation $\pi_{\omega}$ which is the end vertex of the walk corresponding to $\omega$. E.g. the word 2, 3, 3, 1, 2 corresponds to the walk 1234, 1324, 1342, 1324, 3124, 3214 in $W B_{4}$, i.e., $\pi_{2,3,3,1,2}=3214$ (in Figure 1 the coloring is indicated by different gray scales). Maximal chains from id to $\pi$ in $W B_{n}$ are in bijection with the minimum length words $\omega$ such that $\pi=\pi_{\omega}$. Such a minimum length word is known as a reduced decomposition or a reduced word of $\pi$. The permutation 3214 has two reduced words 2,1,2 and 1,2,1.

A pseudoline is a curve in the Euclidean plane whose removal leaves two unbounded regions. An arrangement of pseudolines is a family of pseudolines with the property that each pair of pseudolines has a unique point of intersection where the two pseudolines cross. In a partial arrangement we do not require that every pair of pseudolines has a crossing, i.e., we allow parallel lines. In the case of pseudolines the relation 'parallel' need not be transitive. An arrangement is simple if no three pseudolines have a common point of intersection. An arrangement partitions the plane into cells of dimensions 0,1 or 2 , the vertices, edges and faces of the arrangement. Let $F$ be an unbounded face of arrangement $\mathcal{A}$, call $F$ the northface and let $F^{o}$ be $F$ together with an orientation of the boundary path of $F$. The pair $\left(\mathcal{A}, F^{o}\right)$ is a marked arrangement. Two marked arrangements are isomorphic if there is an isomorphism of the induced cell decompositions of the plane respecting the oriented marking faces. We denote as arrangement an isomorphism class of simple marked arrangements of pseudolines. Similarly a partial arrangement is an isomorphism class of simple marked partial arrangements of pseudolines.

Goodman and Pollack [8] described a one-to-many correspondence from arrangements to reduced decompositions of $w_{0}$ (in this context the name simple allowable sequence is used for these objects). We sketch the connections which carry through to partial arrangements and general reduced decompositions.

Let $(\mathcal{A}, F)$ be a marked partial arrangement of $n$ lines, specify points $x \in F$ and $\bar{x}$ in the complementary face $\bar{F}$ of $F$. A sweep of $\mathcal{A}$ is a sequence $c_{0}, c_{1}, \ldots c_{r}$, of curves from $\bar{x}$ to $x$ which avoid vertices of the arrangement and such that between two consecutive curves $c_{i}$ and $c_{i+1}$ there is exactly one vertex of the arrangement and every vertex of $\mathcal{A}$ is between two curves. An example of a sweep is shown in Figure 2.

Label the lines of $\mathcal{A}$ such that curve $c_{0}$ oriented from $\bar{x}$ to $x$ crosses them in the order $1,2, \ldots, n$. Traversing curve $c_{i}$ from $\bar{x}$ to $x$ we meet the lines of $\mathcal{A}$ in some order. Since each line is met by $c_{i}$ exactly once, the order of the crossings corresponds to a permutation $\pi_{i}$ of $[n]$. If in the arrangement each pair of lines crosses exactly once, then $r=\left(\begin{array}{l}n \\ 2\end{array}\right)$ and $\pi_{r}=w_{0}$. The sequence $\pi_{0}, \ldots, \pi_{r}$ of permutations is a simple allowable sequence or in our terminology a reduced word of $w_{0}$. In the example of Figure 2 we obtain the reduced word $1,2,3,1,2,1$. In general an arrangement $(A, F)$ has various sweeps leading to different reduced words. In our example $1,2,1,3,2,1$ is another sweep.

Conversely a reduced word corresponds to a unique (up to isomorphism) simple marked partial arrangement. A nice construction of an arrangement corresponding to a reduced word is the wiring diagram of Goodman [7]. Let $\omega$ be a reduced word. Start drawing $n$ horizontal lines called wires and vertical lines $p_{0}, \ldots, p_{r}$. Between $p_{i}$ and $p_{i+1}$ draw a X shaped cross between wires $\omega_{i}$ and $\omega_{i}+1$ (wires are counted from bottom to top). 


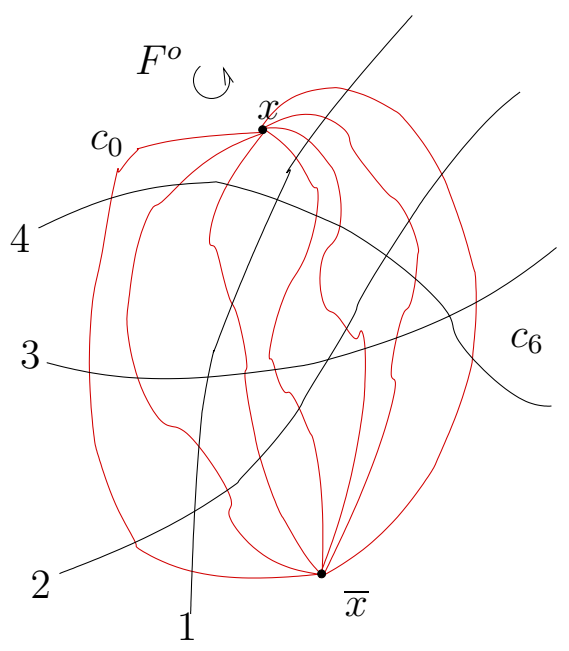

Figure 2: A sweep for arrangement $\mathcal{A}$

Pseudoline $l_{i}$ starts on wire $i$ moves to the right and whenever it meets a cross it changes to the other wire incident to the cross. The construction is illustrated in Figure 3.

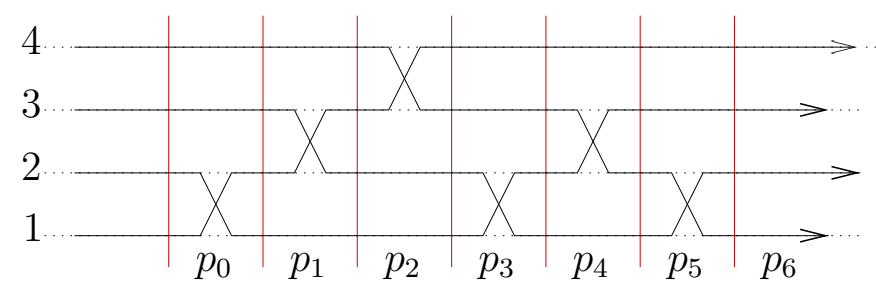

Figure 3: A wiring diagram for the word 1,2,3,1,2,1

Let $\omega=\omega_{1}, \omega_{2}, \ldots, \omega_{r}$ be a reduced word. If $\left|\omega_{i}-\omega_{i+1}\right| \geq 2$, in other words, if the crossings corresponding to $\omega_{i}$ and $\omega_{i+1}$ in the wiring diagram of $\omega$ do not share a line, then the word $\omega^{\prime}$ obtained from $\omega$ by exchange of $\omega_{i}$ and $\omega_{i+1}$ is a reduced word corresponding to the same arrangement. Words $\omega$ and $\omega^{\prime}$ over $N$ are called elementary equivalent if $\omega^{\prime}$ is obtained from $\omega$ by a sequence of transpositions of adjacent letters $\omega_{i}$ and $\omega_{i+1}$ with $\left|\omega_{i}-\omega_{i+1}\right| \geq 2$. This results in the following proposition which is a restatement of classical results of Tits and Ringel, see [1, pp 262-269] for exact references.

Proposition 1. Two reduced words are elementary equivalent iff they correspond to the same isomorphism class of simple marked partial arrangements.

We now come back to the mapping from words $\omega$ over $N$ to permutations $\pi_{\omega}$ in $S_{n}$. It is natural to ask for conditions on $\omega$ and $\omega^{\prime}$ such that they represent the same permutation $\pi_{\omega}=\pi_{\omega^{\prime}}$. The full answer to this question is provided by the Coxeter relations. $\omega$ and $\omega^{\prime}$ represent the same permutation if $\omega$ can be transformed into $\omega^{\prime}$ by a sequence of 
transformations (moves) of the form

$$
\begin{aligned}
i, i & \longleftrightarrow \\
i, j & \longleftrightarrow j, i \\
i, i+1, i & \longleftrightarrow i+1, i, i+1
\end{aligned} \quad|i-j| \geq 2
$$

We call two words equivalent iff they are related by a sequence of moves of type COX 1 and COX 2. Equivalence between $\omega$ and $\omega^{\prime}$ is denoted by $\omega \sim \omega^{\prime}$. With this definition the equivalence class of a reduced word $\omega$ is the set of all reduced words representing the same permutation $\pi_{\omega}$.

\subsection{Young Tableaux, Point Sets and Skeletons}

Let $\lambda$ be a partition of $n=|\lambda|$ with parts $\lambda_{1} \geq \lambda_{2} \geq \ldots \geq \lambda_{m}$. With $\lambda$ we associate a Ferrer's diagram with $\lambda_{i}$ cells in the $i$ th row, see Fig. 4 . We refer to the cells of a diagram

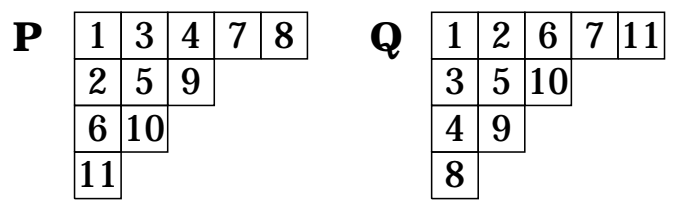

Figure 4: Two standard Young tableaux $\mathbf{P}$ and $\mathbf{Q}$ of shape $\lambda=(5,3,2,1)$.

in matrix notation, rows are numbered from top to bottom, columns from left to right and cell $(i, j)$ is the cell in row $i$ and column $j$. A tableau $\mathbf{T}$ of shape $\lambda$ is an assignment of numbers to the cells of the diagram of $\lambda$. The shape of a tableau $\mathbf{T}$ is denoted $\lambda(\mathbf{T})$. The content cont $(\mathbf{T})$ of tableau $\mathbf{T}$ is the set of entries of cells of $\mathbf{T}$. A tableau $\mathbf{T}$ is a Young tableau if the entries strictly increase in rows and columns. A Young tableau of shape $\lambda$ and content $\{1, \ldots,|\lambda|\}$ is a standard Young tableau, see Fig. 4.

The bijection of the following Proposition is known as the Robinson-Schensted correspondence. This correspondence is the starting point of much combinatorial work on Young tableaux. We refer to $[6,16,18]$ for more comprehensive treatments of this topic.

Proposition 2. There is a bijection between the permutations of $\{1, \ldots, n\}$ and pairs $(\mathbf{P}, \mathbf{Q})$ of standard Young tableaux of the same shape and $|\lambda(\mathbf{P})|=n$.

A set $X$ of points in $\mathbb{R}^{2}$ is said to be in 'general position' if no two points have the same $x$ - or $y$-coordinate. There is a natural mapping from permutations $\{1, \ldots, n\}$ to point sets, with $\pi$ associate $X_{\pi}=\left\{\left(i, \pi_{i}\right): i=1, \ldots, n\right\}$. Via this mapping the following Proposition specializes to the Robinson-Schensted correspondence.

Theorem 1. There is a bijection between $n$ element point sets $X$ in $\mathbb{R}^{2}$ which are in general position and pairs $(\mathbf{P}, \mathbf{Q})$ of Young tableaux of the same shape, with $|\lambda(\mathbf{P})|=n$, $\operatorname{cont}(\mathbf{P}(X))=\{y:(x, y) \in X\}$ and $\operatorname{cont}(\mathbf{Q}(X))=\{x:(x, y) \in X\}$. 
It is possible to remove the 'general position assumption' and even extend Theorem 1 to the case of a multiset $X$, in that case the tableaux corresponding to $X$ have multiple entries and only remain weakly increasing. Basically, this is the extension of the RobinsonSchensted correspondence to two line arrays due to Knuth [10]. The proof given below follows the ideas developed by Viennot in [17, 18]. Algorithmic consequences of the planarization have been obtained in [4], a comprehensive exposition of Viennot's approach is given by Wernisch [19].

Define the shadow of a point $p=(x, y)$ as the set of all points $(u, v)$ dominating $p$, i.e., points with $u>x$ and $v>y$. For a set $E \subseteq X$ of points, the shadow of $E$ is the union of the shadows of the points of $E$, i.e., the set of all points dominating at least one point of $E$ (see the shaded region in Fig. 5).

The jump line, $L(E)$, of a point set $E$ is the topological boundary of the shadow of $E$. The unbounded half lines of jump lines are the outgoing lines, they are specified as right and top. The jump line $L(E)$ of a set $E$ of points is a downward staircase with some points of $E$ in its lower corners.

The dominance relation induces a partial ordering on $E$, in the terminology of partial orders the points of $A=E \cap L(E)$ are the antichain of minimal elements of $E$. The points in the upper-right corners of the jump-line are the skeleton points or skeleton $S(A)$ of the antichain $A$. Formally, if $\left(x_{1}, y_{1}\right), \ldots,\left(x_{k}, y_{k}\right)$ are the points of $A$ ordered by increasing $x$-coordinate then $S(A)$ contains the points $\left(x_{2}, y_{1}\right), \ldots,\left(x_{k}, y_{k-1}\right)$. Hence, $A$ has exactly $|A|-1$ skeleton points (see Fig. 5).

The minimal elements of a point set $X$ form an antichain $A$ such that the rest $X \backslash A$ lies completely in the shadow of $A$. Hence, by removing $A$ and treating $X \backslash A$ in the same way, we recursively obtain the canonical antichain partition $A=A_{0}, \ldots, A_{\lambda-1}$ with non-intersecting jump lines $L\left(A_{i}\right), 0 \leq i<\lambda$, which will be called the layers $L_{i}(X)$ of $X$. The skeleton of $X$, denoted by $S(X)$, is defined as the union of the skeletons $S\left(A_{i}\right)$, $0 \leq i<\lambda$. Since, as noted above, layer $L_{i}(X)$ has $\left|A_{i}\right|-1$ skeleton points the size of $S(X)$ is $|X|-\lambda$. A picture of a point set $X$, its skeleton $S(X)$, its antichain layer partition, and the shadow of antichain $A_{2}$ is shown in Fig. 5.

One of the properties that seem to lie behind the usefulness of skeletons is the fact that it is possible to reconstruct $X$ from $S(X)$ with a small amount of additional information. Let $x_{\max }$ be the maximal $x$-coordinate of points in $X$, and let $y_{\max }$ be defined analogously. Then the right marginal points $M_{R}(X)$ of $X$ are the points $\left(x_{\max }+1, y_{1}\right), \ldots,\left(x_{\max }+\lambda, y_{\lambda}\right)$, where $\lambda$ is the number of layers of $X$ and $y_{1}, \ldots, y_{\lambda}$ are the $y$-coordinates of the right outgoing lines of the layers ordered increasingly (see Fig. 6). Assuming $x_{1}, \ldots, x_{\lambda}$ to be the $x$-coordinates of the top outgoing lines of the layers in increasing order the top marginal points $M_{T}(X)$ of $X$ are $\left(x_{1}, y_{\max }+1\right), \ldots,\left(x_{\lambda}, y_{\max }+\lambda\right)$ (see Fig. 6). With $M(X)$ we denote the marginal points of $X$, i.e., $M(X)=M_{R}(X) \cup M_{T}(X)$.

For a point set $X$ let $-X$ be the set containing $(-x,-y)$ iff $X$ contains $(x, y)$. Define the left-down skeleton $S_{\swarrow}(X)$ as $-S(-X)$. The same result is obtained by defining the left-down shadow of a point $p$ as the set of points dominated by $p$ and defining the leftdown versions of jump-lines, layers and the skeleton in analogy to the definition based on the shadow of a point. 


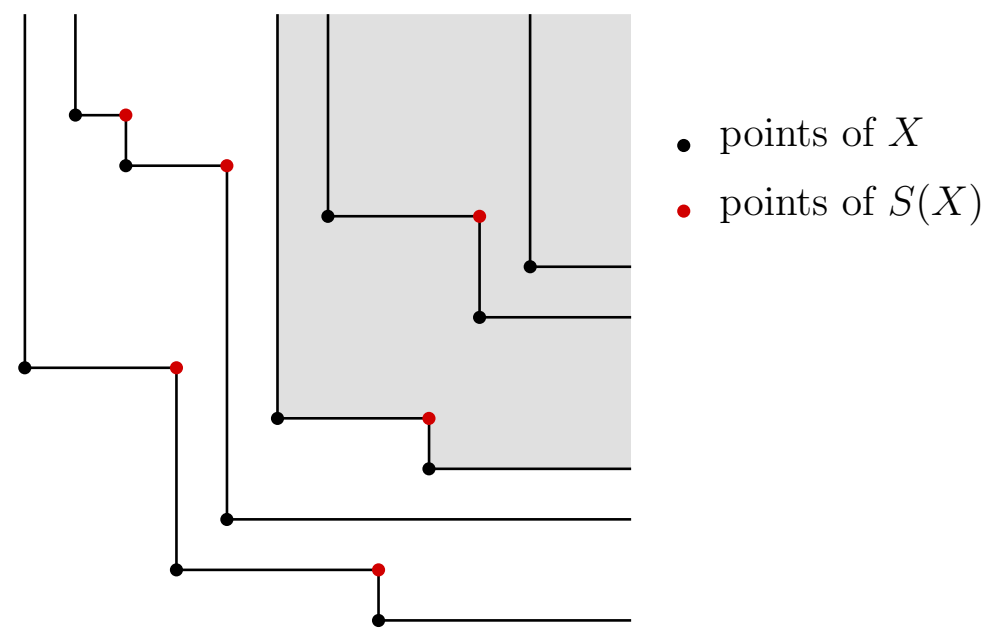

Figure 5: Point set $X$, its skeleton, and the shadow of layer $L_{2}(X)$.

Lemma 1. A point set $X$ is the left-down skeleton of the skeleton $S(X)$ enhanced by the marginal points of $X$, i.e., $X=S_{\swarrow}(S(X) \cup M(X))$.

Let $S^{k}(X)=S\left(S^{k-1}(X)\right)$ denote the $k$ fold application of $S$ to a point set $X$. Since $|S(X)|<|X|$ there is a $m$ such that $S^{m}(X)=\emptyset$, let $\mu(X)$ be the minimum such $m$. Also let $\lambda_{i}(X), 0 \leq i<\mu(X)$, denote the number of layers of $S^{i}(X)$.

Lemma 2. Let $X$ be a planar point set and $\lambda_{i}=\lambda_{i}(X)$ then $\lambda_{0} \geq \lambda_{1} \geq \cdots \geq \lambda_{\mu-1}>0$, and $\left|S^{k}(X)\right|=\sum_{k \leq i<\mu} \lambda_{i}$. In particular $\lambda=\left(\lambda_{0}, \lambda_{1}, \ldots, \lambda_{\mu(X)-1}\right)$ is a partition of $n$.

Proof. We show that $\left(\lambda_{i}\right)$ is a decreasing sequence: By Lemma 1, the number of antichains in a minimal antichain partition of $S(X) \cup M(X)$ is the same as $\lambda_{0}$, the size of the canonical antichain partition of $X$. Hence, $\lambda_{1}(X)$, the size of a minimal antichain partition of $S(X)$ is at most $\lambda_{0}$. The same argument shows the other inequalities. The claim on the size of $S^{k}(X)$ follows by induction from $|S(X)|=|X|-\lambda_{0}(X)$ and its immediate consequence $\left|S^{k+1}(X)\right|=\left|S^{k}(X)\right|-\lambda_{k}(X)$.

We are ready now to describe the bijection of Theorem 1 . With a planar set $X$ of $n$ points we associate two tableaux $\mathbf{P}(X)$ and $\mathbf{Q}(X)$ (the $\mathbf{P}$ - and $\mathbf{Q}$-symbol of $X$ ) in the following way. The $k$-th row of $\mathbf{P}(X), k \geq 0$, are the $y$-coordinates of the right outgoing lines of $S^{k}(X)$ in increasing order. The $k$-th row of $\mathbf{Q}(X), k \geq 0$, are the $x$-coordinates of the top outgoing lines of $S^{k}(X)$ in increasing order. As an example compare the outgoing lines of the first two layers of Fig. 7 with the first two rows of the Young tableaux in Fig. 4. According to Lemma 2, $\mathbf{P}(X)$ and $\mathbf{Q}(X)$ have $\lambda_{i}(X)$ cells in their $i$-th row and $|X|$ cells altogether. Hence, the shape of $\mathbf{P}(X)$ and $\mathbf{Q}(X)$ is the diagram of a partition, moreover, the shapes of $\mathbf{P}(X)$ and $\mathbf{Q}(X)$ are equal and $\operatorname{cont}(\mathbf{P}(X))=\{y:(x, y) \in X\}$ and $\operatorname{cont}(\mathbf{Q}(X))=\{x:(x, y) \in X\}$.

It remains to show that the entries in the cells of the symbols increase along rows and columns. For the rows this is true by construction. For the increase in the columns of $\mathbf{P}$ 


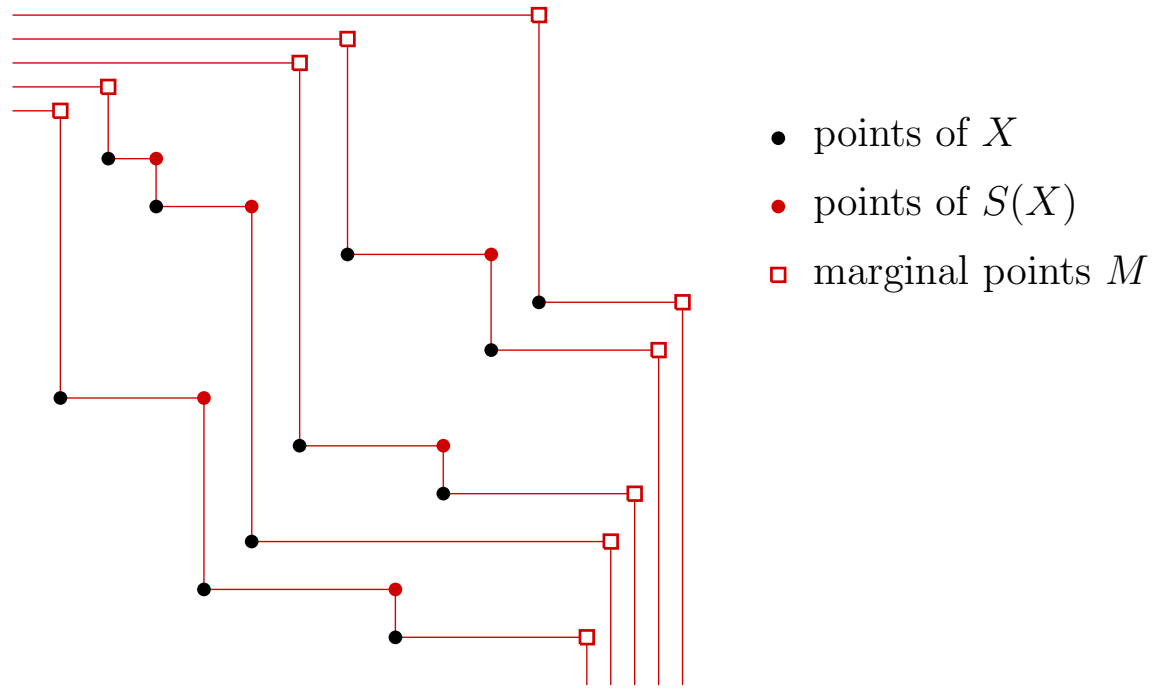

Figure 6: $X$ is the left-down skeleton of $S(X) \cup M(X)$.

we claim that the right outgoing line of layer $L_{j}(X)$ lies below that of the corresponding layer $L_{j}(S(X))$ in the skeleton, i.e., that $\mathbf{P}(0, j) \leq \mathbf{P}(1, j)$. Consider a skeleton point $s$ of height $j$ in the dominance order of $S(X)$. Since a layer of $X$ can only contain one point from a chain in $S(X)$ we conclude that $s$ belongs to some layer $L_{i}(X)$ with $i \geq j$. Hence, $L_{j}(S(X))$ lies in the shadow of $L_{j}(X)$ and its right outgoing line must lie above that of $L_{j}(X)$. Induction implies that $\mathbf{P}(X)$ is a Young tableaux.

The same property for the Q-symbol follows from an important symmetry in the two symbols of a point set. Let the inverse $X^{-1}$ of $X$ be the point set obtained from $X$ by the transposition $(x, y) \rightarrow(y, x)$, i.e., by reflection on the diagonal line $x=y$. The following proposition (Schützenberger) is immediate from the construction.

Proposition 3. The two symbols of the inverse $X^{-1}$ of a point set $X$ are $\mathbf{P}\left(X^{-1}\right)=$ $\mathbf{Q}(X)$ and $\mathbf{Q}\left(X^{-1}\right)=\mathbf{P}(X)$.

We conclude this subsection with the proof that $X \leftrightarrow(\mathbf{P}, \mathbf{Q})$ is a bijection. By Lemma $1 X$ is determined by $S(X)$ and the sets of right and top marginal points, $M_{R}(X)$ and $M_{T}(X)$. The right marginal points are obtained from the first row of $\mathbf{P}(X)$ and the top marginal points from the first row of $\mathbf{Q}(X)$. If we delete the fist row from $\mathbf{P}(X)$ and $\mathbf{Q}(X)$ we are left with the $\mathbf{P}$ and $\mathbf{Q}$ symbols of $S(X)$. With induction this shows that $X$ can be reconstructed from $(\mathbf{P}(X), \mathbf{Q}(X))$. The same construction allows to associate a point set with any pair $(\mathbf{P}, \mathbf{Q})$ of Young tableaux of the same shape.

For more complete exposition of this planarized correspondence and its consequences the reader is referred to Viennot [18] and Wernisch [19]. 


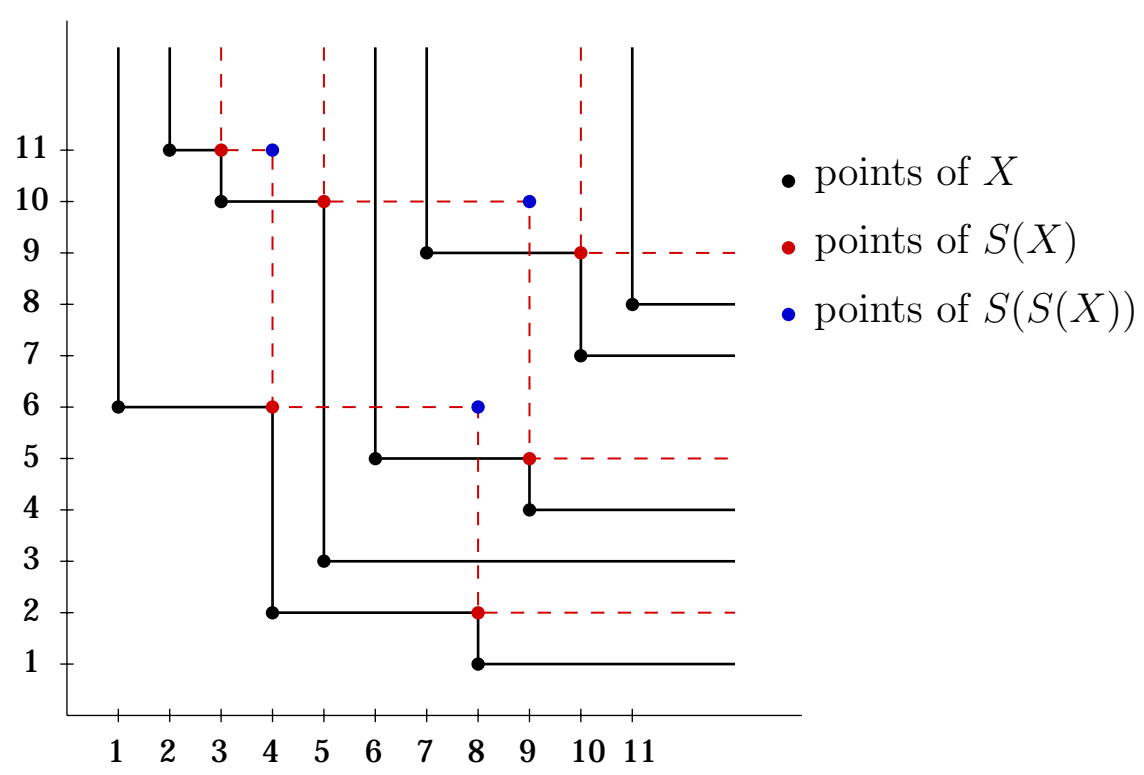

Figure 7: The first two skeletons $S(X)$ and $S^{2}(X)$ of $X$.

\subsection{The Correspondence of Edelman and Greene}

The statement of the correspondence of Edelman and Greene, Proposition 4 is surprisingly similar to the Robinson-Schensted correspondence, Theorem 1.

To state the proposition we need to define the reading $(\mathbf{T})$, of a Young tableau $\mathbf{T}$ as the word obtained by concatenating the rows of $\mathbf{T}$ from bottom to top. For example the reading of the tableau $\mathbf{P}$ of Fig. 4 is the concatenation of $(11)(6,10)(2,5,9)(1,3,4,7,8)$, i.e., reading $(\mathbf{P})=11,6,10,2,5,9,1,3,4,7,8$.

Proposition 4 (Edelman and Greene). There is a bijection between reduced words $\omega$ of permutations in $S_{n}$ and pairs $(\mathbf{P}, \mathbf{Q})$ of Young tableaux of the same shape such that $\mathbf{Q}$ is standard, $|\lambda(\mathbf{Q})|=$ length $(\omega), \operatorname{cont}(\mathbf{P}) \subseteq\{1, \ldots, n-1\}$ and the reading of $\mathbf{P}$ is a reduced word equivalent to $\omega$.

To prepare for our planarized proof of the theorem we extend the notions of words and reduced words. Let $i_{1}<i_{2} \ldots<i_{m}$ be positive integers, a sequence $\omega=\omega_{i_{1}}, \omega_{i_{2}}, \ldots, \omega_{i_{m}}$ with letters $\omega_{i_{j}}$ in the alphabet $N=\{1, \ldots, n-1\}$ will be called a quasi-word. Sometimes it is appropriate to code a quasi-word in two lines, where the top line carries the indices and the bottom line the letters, e.g., $\left(\begin{array}{c}1,3,6,7,8 \\ 2,3,2,1,3\end{array}\right)$. The word obtained from the quasi-word $\omega$ by reindexing $i_{j} \rightarrow j$ is called the normalized word corresponding to $\omega$. If the normalized word of $\omega$ is a reduced word we call $\omega$ a reduced quasi-word.

With a quasi-word $\omega$ we associate a switch diagram as shown in Fig. 8. Begin with $n$ horizontal lines at unit distance, with wire $i$ we denote the $i$-th of these lines counted from bottom to top. With the letter $\omega_{i_{j}}$ of $\omega$ we associate a switch $\left[i_{j}, \omega_{i_{j}}\right]$ at $x$-coordinate $i_{j}$ connecting wires $\omega_{i_{j}}$ and $\omega_{i_{j}}+1$. Note the similarity of this construction to the wiring diagram of Subsection 2.1. Occasionally we use the notation $\omega_{X}$ and $X_{\omega}$ to go from a 
quasi-word $\omega$ to the associated set $X$ of switches and back. In order to have this natural bijection $\omega_{X} \leftrightarrow X_{\omega}$ we make the following general position assumption: A set of switches never contains two switches above each other, i.e., with the same first coordinate. A switch diagram $X$ is normalized iff $\omega_{X}$ is a normalized word, i.e., if the indices of switches are $1,2, \ldots,|X|$.

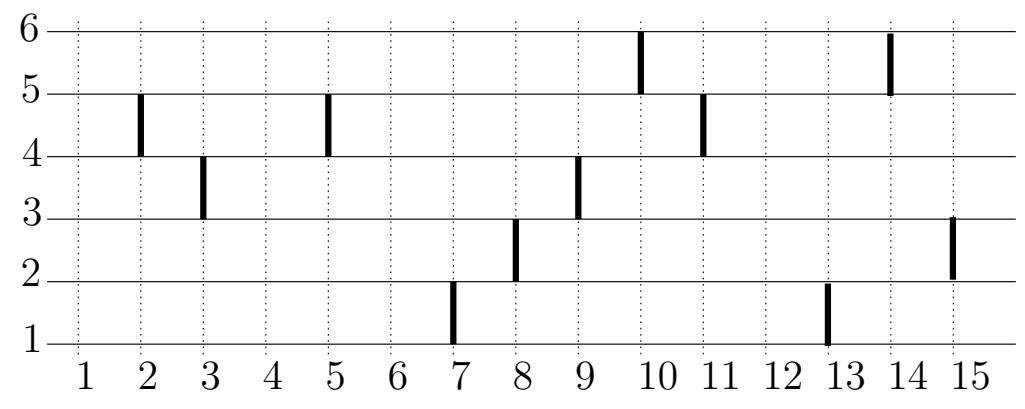

Figure 8: A switch-diagram for the quasi-word $\omega=\left(\begin{array}{l}2,3,5,7,8,9,10,11,13,14,15 \\ 4,3,4,1,2,3,5,4,1,5,2\end{array}\right)$

In analogy to the terminology introduced for point sets we define shadows, jump-lines, layers and skeletons for switch diagrams.

The base point of a switch $s=[i, w]$ the lower end point $(i, w)$ of $s$ and the shadow of $s$ is the region of all points $(u, v)$ which dominate the base point of $s$, i.e., the set of all point which are right and up of the base point. The shadow of a set of switches is the union of the shadows of switches in the set.

The jump line, $L(E)$, of a set $E$ of switches is the topological boundary of the shadow of $E$. The unbounded half lines of jump lines are the outgoing lines, they are specified as right and top.

The jump line $L(E)$ of a set $E \subseteq X$ of switches is a downward staircase. Some switches of $E$ define corners of $L(E)$, they are said to be taken by the jump line, some other switches only have their base point on $L(E)$, they are said to be touched by the jump line. Let $T(E)$ be the set of switches in $E$ which are taken or touched by the jump line of $E$. Note that $T(E)$ corresponds to an decreasing subword of the quasi-word $\omega_{E}$. In the example of Fig. 8 and 9 the decreasing quasi-word corresponding to $T\left(E_{\omega}\right)$ is $\left(\begin{array}{c}2,3,7,13 \\ 4,3,1,1\end{array}\right)$. On switches we define an order relation, for $s=[i, w]$ and $s^{\prime}=\left[i^{\prime}, w^{\prime}\right]$ we say $s^{\prime}$ dominates $s$ if $i<i^{\prime}$ and $w<w^{\prime}$. This allows us to speak of chains and antichains of switches. Note that $T(E)$ is just the antichain of minimal switches in the dominance order induced by E.

Let $A$ be an antichain of switches, i.e., $A=T(A)$. The jump line $L(A)$ has a corner above each but the first of the switches taken by the jump-line, let $C(A)$ be the set of these corners and let $D(A)$ be the set of upper ends of the switches touched by $L(A)$. The set of skeleton switches of the jump line $L(A)$ is the set of switches with base point in $C(A) \cup D(A)$ (see Fig. 9). The set of skeleton switches of $A$ is denoted as $S(A)$. We emphasize two important properties of the skeleton of an antichain:

- If $A$ is antichain of switches then $|S(A)|=|A|-1$. 
- The skeleton switches $S(A)$ of an antichain $A$ are again an antichain.

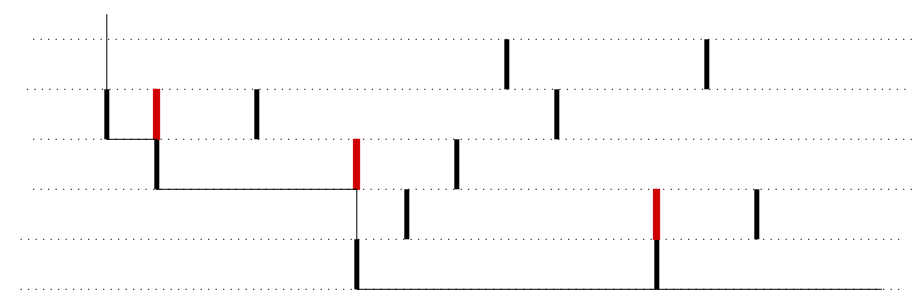

\section{| Switches of $E$ | Skeleton switches}

Figure 9: A set $E$ of switches, its jump line and the skeleton switches of $T(E)$

For a set $X$ of switches every switch $s \in X \backslash T(X)$ lies completely in the shadow of $T(X)$. Hence, by removing $A_{0}=T(X)$ and treating $X \backslash T(X)$ in the same way, we recursively obtain a partition $A_{0}, A_{1}, \ldots, A_{\lambda-1}$ of $X$ into antichains. As in the case of points this is the partition by height of the dominance order, we call it the canonical partition of $X$. The canonical partition is a minimal partition into antichains.

The jump lines $L\left(A_{i}\right), 0 \leq i<\lambda$, are pairwise non-intersecting, they will be called the layers $L_{i}(X)$ of $X$. The skeleton of $X$, denoted by $S(X)$, is defined as the union of the skeletons $S\left(A_{i}\right), 0 \leq i<\lambda$. Since, as noted above, layer $L_{i}(X)$ has $\left|A_{i}\right|-1$ skeleton points the size of $S(X)$ is $|X|-\lambda$. An example for a set of switches, its layers and the skeleton is given in Fig. 10.

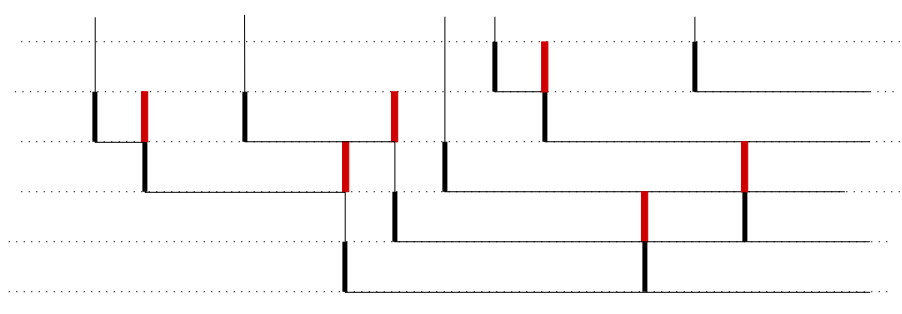

Figure 10: Layers and skeleton of the set $X$ of Fig. 9.

Let $S^{k}(X)=S\left(S^{k-1}(X)\right)$ denote the $k$ fold application of $S$ to a set $X$ of switches. Since $|S(X)|<|X|$ there is an $m$ such that $S^{m}(X)=\emptyset$, let $\mu(X)$ be the minimum such $m$. Also let $\lambda_{i}(X), 0 \leq i<\mu(X)$, denote the number of layers of $S^{i}(P)$.

Lemma 3. Let $X$ be set of switches and $\lambda_{i}=\lambda_{i}(X)$ then $\lambda_{0} \geq \lambda_{1} \geq \cdots \geq \lambda_{\mu(X)-1}>0$, and $\left|S^{k}(X)\right|=\sum_{k \leq i<\mu(X)} \lambda_{i}$. In particular $\lambda=\left(\lambda_{0}, \lambda_{1}, \ldots, \lambda_{\mu(X)-1}\right)$ is a partition of $|X|$.

Proof. We show that $\left(\lambda_{i}\right)$ is a decreasing sequence: Let $A_{0}, \ldots, A_{\lambda_{0}-1}$ be the layers of $X$. Recall that $S\left(A_{j}\right)$ is an antichain and $\bigcup_{j} S\left(A_{j}\right)=S(X)$, hence, $S\left(A_{0}\right), \ldots, S\left(A_{\lambda_{0}-1}\right)$ is a partition into antichains. Since $\lambda_{1}$ is the size of a minimal partition of $S(X)$ into antichains we have $\lambda_{0} \geq \lambda_{1}$. The same argument shows the other inequalities. The claim on the size of $S^{k}(X)$ follows by induction from $|S(X)|=|X|-\lambda_{0}(X)$ and its immediate consequence $\left|S^{k+1}(X)\right|=\left|S^{k}(X)\right|-\lambda_{k}(X)$. 


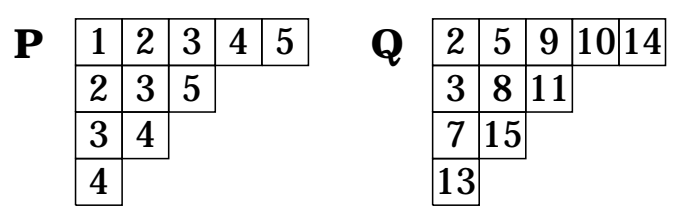

Figure 11: The two tableaux $\mathbf{P}$ and $\mathbf{Q}$ associated with the quasi-word $\omega$ from Fig. 8 .

Now we are ready to describe a mapping from a switch diagram $X$ with $n$ switches to a pair $(\mathbf{P}(X), \mathbf{Q}(X))$ of tableaux. The $k$-th row of $\mathbf{P}(X), k \geq 0$, are the numbers of the wires of the right outgoing lines of $S^{k}(X)$ in increasing order. The $k$-th row of $\mathbf{Q}(X), k \geq 0$, are the indices of the top outgoing lines of $S^{k}(X)$ in increasing order. As an example compare the outgoing lines of the first layer of Fig. 9 with the first row of the two tableaux in Fig. 11. We note some properties of the two tableaux:

- The shapes of $\mathbf{P}(X)$ and $\mathbf{Q}(X)$ are equal.

- The shape of $\mathbf{P}(X)$ and $\mathbf{Q}(X)$ is the diagram of the partition $\left(\lambda_{0}, \lambda_{1}, \ldots, \lambda_{\mu(X)-1}\right)$ of $|X|$, (Lemma 3).

- The entries of $\mathbf{P}(X)$ and $\mathbf{Q}(X)$ are strictly increasing in every row.

- $\operatorname{cont}(\mathbf{Q}(X))=\{i: s=[i, w] \in X\}$ and $\{w: s=[i, w] \in X\} \subseteq \operatorname{cont}(\mathbf{P}(X))$.

Given these properties the proof that $\mathbf{P}(X)$ and $\mathbf{Q}(X)$ are Young tableaux can be completed by showing that the entries of both tableaux are strictly increasing in columns.

Lemma 4. The jump line $L_{j}(S(X))$ is contained in the shadow of $L_{j}(X)$ and they can only touch in corners.

Proof. Let $s$ be a skeleton switch of layer $j$, i.e., $s \in L_{j}(S(X))$, then $s$ is the highest switch of a chain $s_{1}<s_{2}<\ldots<s_{j}=s$ in $S(X)$. Consider the antichain partition $A_{0}, \ldots, A_{\lambda_{0}-1}$ of $X$. Since $S\left(A_{i}\right)$ is an antichain, for each $i$ the switches of the chain are in the skeleton of different $A_{i}$. Hence, $s=s_{j} \in S\left(A_{k}\right)$ for some $k \geq j$. Switch $s$ can touch $L_{j}(X)$ only if $k=j$ and if $s$ is above a switch $t \in A_{j}$ which is taken by $L_{j}(X)$, i.e., if the base point of $s$ is at a right-down corner of $L_{j}(X)$. Considering the switches of $L_{j}(S(X)$ from left to right it thus follows that $L_{j}\left(S(X)\right.$ and $L_{j}(X)$ can only touch in corners.

It follows that the top outgoing line of $L_{j}(S(X))$ is to the right of the top outgoing line of $L_{j}(X)$ and the right outgoing line of $L_{j}(S(X))$ is above the right outgoing line of $L_{j}(X)$. Hence, $\mathbf{P}(X)$ and $\mathbf{Q}(X)$ are strictly increasing in columns. This completes the proof of the next proposition.

Proposition 5. To every quasi-word $\omega$ of length $r$ the above mapping associates a pair $(\mathbf{P}, \mathbf{Q})$ of Young tableaux of the same shape, with $|\lambda(\mathbf{P})|=r$ and $\operatorname{cont}(\mathbf{Q}(\omega))=\{i$ : $i$ is an index in $\omega\}$ and $\{w: w$ is a letter in $\omega\} \subseteq \operatorname{cont}(\mathbf{P}(\omega))$.

If we restrict this mapping to reduced words, it is the bijection of Edelman and Greene (Prop. 4); this will be shown in the next section. In general different quasi-words can be 
mapped to the same pair of tableaux. The simplest case for this phenomenon is shown in Fig. 12; the words $\omega_{1}=(1,1)$ and $\omega_{2}=(2,1)$ are both mapped to $(\mathbf{T}, \mathbf{T})$ with $\mathbf{T}=1$.
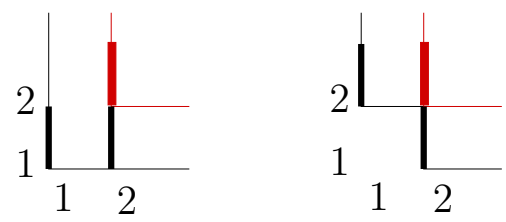

Figure 12: Two switch diagrams associated with the same pair of Young tableaux.

Definition 1. Let $X$ be a set of switches. A bad switch of $X$ is a switch $s=[i, w]$ such that $s \in A_{j}$ implies that $s$ is touched by $L_{j}$ and the upper end $(i, w+1)$ is not on $L_{j+1}(X)$. Set $X$ is good if it contains no bad switch. $X$ is very good if $S^{k}(X)$ is good for $0 \leq k \leq \mu(X)-1$.

In the left example of Fig. 12 switch $[2,1]$ is bad, however, the right set of switches is very good. We are ready now to formulate our main results.

Theorem 2. The mapping $X \rightarrow(\mathbf{P}(X), \mathbf{Q}(X))$ is an injective mapping from very good sets of switches to pairs of Young tableaux of the same shape such that $\operatorname{cont}(\mathbf{Q})=\{i$ : $[i, w] \in X\}$ and $\operatorname{cont}(\mathbf{P})=\{w:[i, w] \in X\}$. In particular, if $X$ is normalized then $\mathbf{Q}(X)$ is standard.

The two tableaux of Fig. 13 show that the mapping $X \rightarrow(\mathbf{P}(X), \mathbf{Q}(X))$ is not a surjection to pairs of Young tableaux with $\mathbf{Q}$ standard and $\operatorname{cont}(\mathbf{P}) \subseteq \mathbb{N}$.

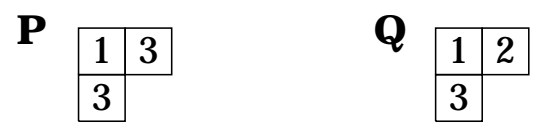

Figure 13: Two tableaux $\mathbf{P}$ and $\mathbf{Q}$ with no associated set $X$ of switches such that $(\mathbf{P}, \mathbf{Q})=$ $(\mathbf{P}(X), \mathbf{Q}(X))$.

So far the only technique to decide whether $(\mathbf{P}, \mathbf{Q})$ is in the image of the mapping is to try to apply the inverse mapping and see if it fails. It would be interesting to find a better characterization of those pairs $(\mathbf{P}, \mathbf{Q})$ which are in the image of the mapping. In other words it would be interesting to understand the bijection hidden in the theorem. From the next theorem it follows that the bijection of Edelman and Greene (Prop. 4) is contained in the more general bijection of Theorem 2 .

\section{Theorem 3.}

(1) If $\omega$ is a reduced quasi-word then $X_{\omega}$ is a very good set of switches.

(2) If $X$ is a very good set of switches, then $\omega_{X} \sim \operatorname{reading}(\mathbf{P}(X))$. 
We indicate how Proposition 4 is obtained from this theorem: If $\omega$ is reduced then $X_{\omega}$ is very good, by (1), hence, $\omega \sim \operatorname{reading}(\mathbf{P})$ by $(2)$. Since they have the same length reading $(\mathbf{P})$ is a reduced word iff $\omega$ is reduced.

The example of Fig. 14 shows that part (2) of the theorem can not be improved to an "if and only if' statement.

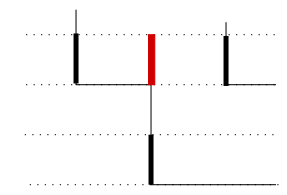

Figure 14: A very good set $X$ of switches such that $\omega_{X}=3,1,3$ is not reduced.

The generalization Proposition 4 provided by Theorem 2 and 3 to is similar to the extension of the Robinson-Schensted correspondence to two line arrays due to Knuth [10].

\section{Proofs of Theorems 2 and 3}

For the proof that the mapping is injective it is convenient to review the construction of the skeleton and the iterated skeletons of switch diagrams. The idea is that we can compute all iterated skeletons in one single sweep from left to right through the diagram. Consider a diagram $X$ and let $s=[t, w]$ be the switch of largest index $t$ in $X$ and $X^{\prime}=X \backslash\{s\}$. Given the canonical partition $A_{0}^{\prime}, A_{1}^{\prime}, \ldots, A_{\lambda^{\prime}-1}^{\prime}$ of $X^{\prime}$ with layers $L_{j}^{\prime}=L\left(A_{j}^{\prime}\right)$ the canonical partition of $X$ is obtained by inserting $s$ into the set $A_{j}^{\prime}$ with $j$ minimal such that the right outgoing line of $L_{j}^{\prime}$ is on a wire $\geq w$. To be more precise, let $S^{\prime}=S\left(X^{\prime}\right)$ and $h_{0}^{\prime}<h_{1}^{\prime}<\ldots<h_{\lambda^{\prime}-1}^{\prime}$ be the wires of the right outgoing lines of the layers of $X^{\prime}$. Skeleton and layers of $X$, i.e., after insertion of $s=[t, w]$, are obtained according to one of the following cases:

(1) If $h_{\lambda^{\prime}-1}^{\prime}<w$ then $A_{\lambda^{\prime}}=\{s\}$, i.e., $s$ generates a new layer which takes $s$. In this case $S(X)=S\left(X^{\prime}\right)$ and the outgoing lines of $X$ are at heights $h_{0}^{\prime}, h_{1}^{\prime}, \ldots, h_{\lambda^{\prime}-1}^{\prime}, w$.

(2) If $h_{j}^{\prime}>w$ and $h_{j+1}^{\prime}<w$ then $A_{j}=A_{j}^{\prime} \cup\{s\}$, i.e., $s$ is taken by layer $j$. In this case a new skeleton switch is created: $S(X)=S\left(X^{\prime}\right) \cup\left\{\left[t, h_{j}^{\prime}\right]\right\}$. The outgoing lines of $X$ are at heights $h_{0}^{\prime}, \ldots, h_{j-1}^{\prime}, w, h_{j+1}^{\prime}, \ldots, h_{\lambda^{\prime}-1}^{\prime}$.

(3) If $h_{j}^{\prime}=w$ and $h_{j+1}^{\prime}=w+1$ then $A_{j}=A_{j}^{\prime} \cup\{s\}, s$ is touched by layer $j$. In this case a new skeleton switch is created: $S(X)=S\left(X^{\prime}\right) \cup\{[t, w+1]\}$. The outgoing lines of $X$ remain at the same heights as those of $X^{\prime}$.

(4) If $h_{j}^{\prime}=w$ and $h_{j+1}^{\prime}>w+1$ then switch $s$ is a bad switch. A new skeleton switch $[t, w+1]$ is created and the outgoing lines of $X$ remain at the same heights as those of $X^{\prime}$.

The new skeleton switch (if there is one) is handled recursively according to the same rules. Note that if $X$ is very good, i.e., rule (4) is never applied, then the horizontal 
segments of jump lines of all iterated skeletons remain on wires containing the base point of a switch. Since a wire which is occupied by a segment of a jump line is occupied by segments of jump lines everywhere to the right we obtain: If $X$ is very good then $\operatorname{cont}(\mathbf{P})=\{w:[i, w] \in X\}$.

We restate the above procedure on the level of the associated tableaux. Let $\left(\mathbf{P}^{\prime}, \mathbf{Q}^{\prime}\right)$ be the tableaux associated with $X^{\prime}$. Let $P_{0}, P_{1}, \ldots, P_{\lambda^{\prime}-1}$ be the rows of $\mathbf{P}^{\prime}$ and recall that $P_{i}$ lists the wires of the right outgoing lines of $S^{i}\left(X^{\prime}\right)$ in increasing order. To avoid special cases we consider $P_{\lambda^{\prime}}$ as an empty row. From the above we obtain by an easy translation that the tableau $\mathbf{P}$ associated with $X=X^{\prime} \cup\{[t, w]\}$ is obtained by the following iteration with initial values $w_{0}=w$ and $i=0$ :

(1) If $w_{i}$ is greater than every entry of $P_{i}$ add $w_{i}$ at the end of this row and stop.

(2) If the least value $x$ in $P_{i}$ with $x \geq w_{i}$ is greater than $w_{i}$, then replace $x$ by $w_{i}$ in this row, let $w_{i+1}=x$ and $i=i+1$, continue with (1).

(3) If the least value $x$ in $P_{i}$ with $x \geq w_{i}$ equals $w_{i}$, then let $w_{i+1}=w_{i}+1$ and $i=i+1$, continue with (1).

This modified-bumping yields the tableau $\mathbf{P}$. The recording tableau $\mathbf{Q}$ is $\mathbf{Q}^{\prime}$ augmented by the unique cell of $\lambda(\mathbf{P}) \backslash \lambda\left(\mathbf{P}^{\prime}\right)$, the entry of this cell is $t$.

Statement (2) from the Theorem (proof follows) tells us that the set of switches of a reduced word is very good. Therefore, case (3) of the above bumping procedure is only applied when $P_{i}$ contains $w_{i}$ and $w_{i}+1$. This shows that the pair of Young tableaux associated to a reduced word $\omega$ by our construction is the same as the pair associated to $\omega$ by the generalized RSK correspondence of Edelman and Greene ([2], Defin.6.21).

\subsection{Proof of Theorem 2}

The proof is by induction on $n$. Let $X$ be a very good set of switches and $X=X^{\prime} \cup\{[t, w]\}$, where $t$ is the largest index of a switch of $X$. Let $(\mathbf{P}, \mathbf{Q})=(\mathbf{P}(X), \mathbf{Q}(X))$. The entry $t$ is in some extremal cell of $\mathbf{Q}$. Say, it is the last cell of row $Q_{k}$ of $\mathbf{Q}$. Let $\mathbf{Q}^{\prime}$ be obtained from $\mathbf{Q}$ by deletion of this cell. Working with rows $P_{k}, P_{k-1} \ldots, P_{0}$ of $\mathbf{P}$ we construct a sequence $s_{j}=\left[t, w_{j}\right]$ of switches with $w_{k}>w_{k-1}>\ldots>w_{0}$. Switch $s_{j}$ will be an element of $\left.S^{j}(X)\right)$. We claim the following:

(a) $w_{0}=w$, i.e., the last switch of $X$ can be reconstructed from $(\mathbf{P}, \mathbf{Q})$.

(b) Via the sequence $s_{k}, \ldots, s_{0}$ of switches the right outgoing lines of $X^{\prime}$ and the iterated skeletons of $X^{\prime}$ are uniquely determined. These outgoing lines determine the Young tableaux $\mathbf{P}^{\prime}=\mathbf{P}\left(X^{\prime}\right)$.

We have assumed that $t$ was the entry of the last cell of row $Q_{k}$ of length $\lambda_{k}$. From the construction of $\mathbf{Q}=\mathbf{Q}(X)$ the top outgoing line at $x$-coordinate $t$ comes from $S^{k}(X)$ and leaves at wire $w_{k}$ where $w_{k}$ is recorded in the last cell of $P_{k}$. Since the jump line $L_{\lambda_{k}-1}\left(S^{k}(X)\right)$ can only have one corner there is a switch $s_{k}=\left[t, w_{k}\right]$ in $S^{k}(X)$. 
When switch $s_{i}=\left[t, w_{i}\right], k \geq i>0$, from $S^{i}(X)$ has been constructed we turn to the construction of $s_{i-1}$ from $S^{i-1}(X)$ below $s_{i}$. Switch $s_{i-1}$ is either touched or taken by its jump line. These two cases can be distinguished by comparing $w_{i}$ to row $P_{i-1}$ :

If switch $s_{i-1}$ is touched, then, since $X$ is very good, there are jump lines of $S^{i-1}(X)$ on wires $w_{i}$ and $w_{i}-1$ at $t$ and to the right of $t$. This happens if $P_{i-1}$ contains entries $w_{i}$ and $w_{i}-1$.

If switch $s_{i-1}$ is taken, then $\left(t, w_{i}\right)$ is a right down corner of a jump line of $S^{i-1}(X)$ which continues to the right at some wire $w_{i-1}<w_{i}$. The value $w_{i-1}$ is the largest entry $x<w_{i}$ of $P_{i-1}$.

In both cases $s_{i-1}=\left[t, w_{i-1}\right]$ with $w_{i-1}$ being the the largest entry $x<w_{i}$ of $P_{i-1}$. In the first case the $(i-1)$-st row $P_{i-1}^{\prime}$ of $\mathbf{P}^{\prime}$ equals $P_{i-1}$. In the second case $P_{i-1}^{\prime}$ is obtained from $P_{i}$ by replacing $w_{i-1}$ by $w_{i}$.

\subsection{Proof of Theorem 3}

The proof of (1) is in two parts.

(a) If $\omega$ is a reduced quasi-word then $X_{\omega}$ is a good set of switches.

(b) If $\omega$ is a reduced quasi-word and $Y=S\left(X_{\omega}\right)$ then $\omega_{Y}$ is again a reduced quasi-word.

Let $\omega$ be a reduced quasi-word. We view the switch diagram $X_{\omega}$ as a wiring diagram, lines enter from the left and at every switch the lines on the wires connected by the switch cross, i.e., both lines change the wire. Since $\omega$ is reduced, any two lines cross at most once. Now assume that $X_{\omega}$ is not good. From this assumption it will follow that there are two lines crossing twice, a contradiction.

Let $s=[t, w]$ be the leftmost bad switch of $X_{\omega}$. Starting from $s$ we define two sequences $a_{w}, a_{w-1}, \ldots$ and $b_{w}, b_{w-1}, \ldots$ of switches. Fig. 15 illustrates the construction which is explained next.

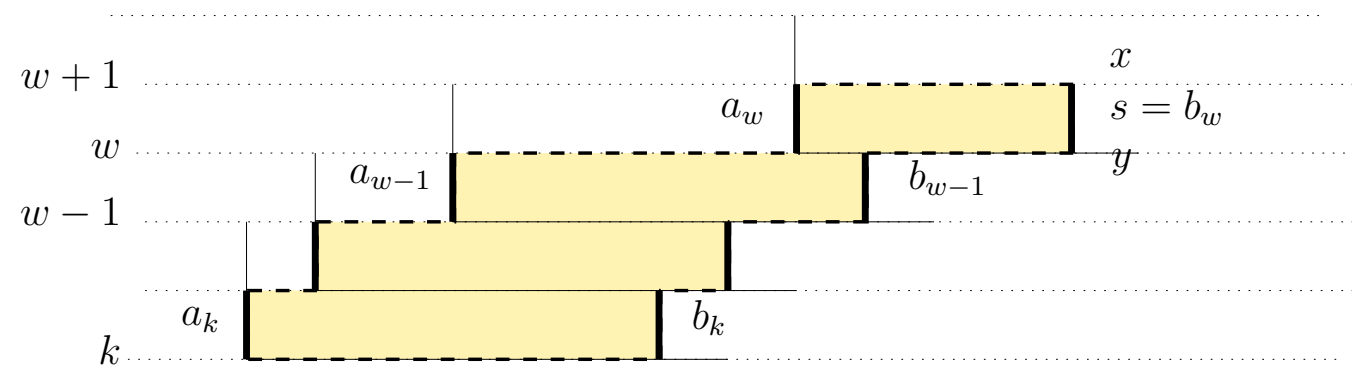

Figure 15: The sequences $a_{w}, a_{w-1}, \ldots$ and $b_{w}, b_{w-1}, \ldots$ and their region.

Let $b_{w}=s$ be the bad switch and $L_{j}$ be the jump line of $X$ touching $s$. Define $a_{w}$ as the switch taken by $L_{j}$ when it comes down to wire $w$. From the choice of $s$ as the first bad switch and the defining property of badness we conclude: 
- Between $a_{w}$ and $b_{w}$ wire $w+1$ is not incident to a switch.

Let $b_{w-1}$ be the rightmost switch connecting to wire $w$ from below but left of $b_{w}$. If $b_{w-1}$ is left of $a_{w}$ then stop the construction. Otherwise, if $b_{w-1}$ is between $a_{w}$ and $b_{w}$ then $b_{w-1}$ is touched by jump line $L_{j-1}$. Let $a_{w-1}$ be the switch taken by $L_{j}$ when it comes down to wire $w$. Again from the choice of $s$ and since every switch belongs to a jump line it follows that:

- Between $a_{w-1}$ and $a_{w}$ wire $w$ is not incident to a switch.

Iterate this construction until it stops at some wire $k \geq 1$. Now consider the two lines $x$ and $y$ crossing at switch $s$. Let $y$ be on wire $w$ to the right of of $s$. Since no switch is connected to wire $i$ between $a_{i-1}$ and $a_{i}, k<i \leq w$, and to wire $w+1$ between $a_{w}$ and $b_{w}=s$. It is easy to trace $y$ backwards and see that $y$ is changing wires at the switches $a_{k}, \ldots, a_{w}, s$. Now consider line $x$. No switch is connected to wire $i$ between $b_{i-1}$ and $b_{i}$, $k<i \leq w$, and between $a_{k}$ and $b_{k}$ from below. Therefore, it is impossible for line $x$ to enter the region between the $a_{i}$ 's and the $b_{i}$ 's (the gray region of Figure 15) from below. Again using that no switch is connected to wire $i$ between $a_{i-1}$ and $a_{i}, k<i \leq w$, it follows, that line $x$ has to use one of the switches $a_{k}, \ldots, a_{w}$. This shows that lines $x$ and $y$ cross twice in contradiction to the assumption that $\omega$ is reduced.

The second part of the proof of (1) is based on the next lemma which will also be central to the proof of part (2) of the theorem.

Lemma 5. If $\omega$ is a good word, $P_{0}$ is the first row of the $\mathbf{P}$-symbol of $\omega$ and $Y=S\left(X_{\omega}\right)$ then the concatenation $\omega_{Y} \circ P_{0}$ is equivalent to $\omega$.

Proof. First note that the right outgoing lines of $X$ and $S(X) \circ P_{0}$ are on the same wires. The right outgoing lines of $X$ are on the wires specified by $P_{0}$. By Lemma 4 the $j$-th jump line of $S(X)$ is above the $j$-th switch of $P_{0}$, hence, it can carry on to contain that switch. Since the switches of $P_{0}$ form a chain they are on different jump lines and $P_{0}$ encodes the wires of the outgoing lines of $S(X) \circ P_{0}$.

The lemma is proved by induction on the length $n$ of $\omega$. Let $X=X_{\omega}$ and $s=[n, w]$ be the last switch of $X$. Let $X^{\prime}=X \backslash\{s\}$ and assume the result for $X^{\prime}$, i.e., $X^{\prime} \sim S\left(X^{\prime}\right) \circ P_{0}^{\prime}$ and the right outgoing lines are on the wires specified by $P_{0}^{\prime}$. We distinguish several cases:

Case 1. If $s$ creates a new highest layer and no skeleton switch. Then $S(X)=S\left(X^{\prime}\right)$ and $P_{0}=P_{0}^{\prime}, w$.

Case 2. If $s$ is touched by its jump line. Let $P_{0}^{\prime}=x_{1}, \ldots, x_{i}, x_{i+1}, \ldots, x_{k}$ with $w=x_{i}$ and since $X$ is good $x_{i+1}=w+1$. In this case $P_{0}=P_{0}^{\prime}$ and the skeleton switch generated by $s$ is $s_{1}=[n, w+1]$. We show that $P_{0}^{\prime}, w \sim(w+1), P_{0}$.

$$
\begin{aligned}
& x_{1}, \ldots, x_{i}, x_{i+1}, \ldots, x_{k}, w \sim \text { (a sequence of COX } 1 \text { moves) } \\
& x_{1}, \ldots, x_{i}, x_{i+1}, w, \ldots, x_{k} \sim \text { (a single COX } 2 \text { move) } \\
& x_{1}, \ldots,(w+1), x_{i}, x_{i+1}, \ldots, x_{k} \sim \quad \text { (a sequence of COX } 1 \text { moves) } \\
& (w+1), x_{1}, \ldots, x_{i}, x_{i+1}, \ldots, x_{k} \text {. }
\end{aligned}
$$


Case 3. If $s$ is taken by its jump line. Again let $P_{0}^{\prime}=x_{1}, \ldots, x_{k}$ and let $x_{i}$ be the first index such that $w<x_{i}$, note that $x_{i-1}<x_{i}-1$. Since $P_{0}=x_{1}, \ldots, x_{i-1}, w, x_{i+1}, \ldots, x_{k}$ and the skeleton switch generated by $s$ is $s_{1}=\left[n, x_{i}\right]$ we have to show that $P_{0}^{\prime}, w \sim x_{i}, P_{0}$.

$$
\begin{aligned}
x_{1}, \ldots, x_{i}, \ldots, x_{k}, w & \sim \quad \text { (a sequence of COX } 1 \text { moves) } \\
x_{1}, \ldots, x_{i}, w, x_{i+1}, \ldots, x_{k} & \sim \quad \text { (a sequence of COX } 1 \text { moves) } \\
x_{i}, x_{1}, \ldots, x_{i-1}, w, x_{i+1}, \ldots, x_{k} . &
\end{aligned}
$$

We complete the proof of (1) by showing that if $\omega$ is a reduced quasi-word and $Y=S\left(X_{\omega}\right)$ then $\omega_{Y}$ is again a reduced quasi-word. Since $\omega_{Y} \circ P_{0}$ is a reduced word for the same permutation as $\omega$ and both words have the same length $\omega_{Y} \circ P_{0}$ is reduced. Since every initial segment of a reduced word is reduced we conclude that $\omega_{Y}$ is reduced.

Iterated application of Lemma 5 shows that a very good word $\omega$ with $\mathbf{P}$-symbol $\mathbf{P}$ is equivalent to $P_{\mu-1} \circ \ldots P_{1} \circ P_{0}=\operatorname{reading}(\mathbf{P})$. This is statement $(2)$ of the theorem.

\section{Concluding Remarks}

Let $\omega$ be a reduced decomposition of $w_{0}$. The $\mathbf{P}$-symbol of $X_{\omega}$ is a Young tableaux with $\left(\begin{array}{l}n \\ 2\end{array}\right)$ entries from $N=\{1, \ldots, n-1\}$. It is easy to see that there is only one such tableau. The shape of this tableau is the staircase shape $\lambda=\{n-1, n-2, \ldots, 1\}$. Therefore $\omega$ is uniquely determined by its Q-symbol. This proves the formula of Stanley shown in the introduction.

Edelman and Greene have also given nice descriptions of the two tableaux associated to the reverse $w_{k}, w_{k-1}, \ldots, w_{1}$ and the reflection $n-w_{1}, n-w_{2}, \ldots, n-w_{k}$ of a reduced word $\omega=w_{1}, w_{2}, \ldots, w_{k}$. These descriptions involving Schützenberger's slide and evacuation operation and transposes largely resemble the corresponding statements for permutations. We need some preparatory definitions to state these results.

The transpose $\mathbf{T}^{T}$ of tableau $\mathbf{T}$ is obtained by reflecting $\mathbf{T}$ on the diagonal $x=-y$, just as matrices are transposed. The slide $s(\mathbf{T})$ of a Young tableau $\mathbf{T}$ is obtained by deleting the corner cell $c=(0,0)$ and then filling the gap. To fill a gap in cell $c=(i, j)$ look at cells $(i+1, j)$ and $(i, j+1)$, i.e., at the cell below and that to the right of $c$. If both contain elements move the smaller one to $c$. If only one contains an element, take

that. The new gap is treated similarly until there is no element below and to the right of the cell with the gap, i.e., until the gap has moved into an upper corner cell. If all entries of $\mathbf{T}$ are different this process is well defined and actually yields a Young tableau. The evacuation tableau evac $(\mathbf{T})$ of a standard Young tableau $\mathbf{T}$ is the recording tableau for the sequence of shapes obtained by iterating the slide operation, i.e., the recording tableau for the sequence

$$
\emptyset, s^{n-1}(\mathbf{T}), s^{n-2}(\mathbf{T}), \ldots, s^{2}(\mathbf{T}), s(\mathbf{T}), \mathbf{T}
$$


Proposition 6. Let $\pi$ be a permutation and $\bar{\pi}$ its reverse then

$$
(\mathbf{P}(\bar{\pi}), \mathbf{Q}(\bar{\pi}))=\left(\mathbf{P}(\pi)^{T}, \operatorname{evac}(\mathbf{Q}(\pi))^{T}\right)
$$

Proposition 7. Let $\omega$ be a reduced word with reverse $\bar{\omega}$ and reflection $\widehat{\omega}$ then

$$
(\mathbf{P}(\bar{\omega}), \mathbf{Q}(\bar{\omega}))=\left(\mathbf{P}(\omega)^{T}, \operatorname{evac}(\mathbf{Q}(\omega))^{T}\right) \quad \text { and } \quad \mathbf{Q}(\widehat{\omega})=\mathbf{Q}(\omega)^{T} .
$$

A planarized proof for Proposition 6 was given by Wernisch [19]. The main tool for his proof is a lemma showing that the skeleton operator $S_{\nwarrow}$ with respect to north-west shadows and the standard skeleton operator $S$, which is defined with respect to northeast shadows, commute. He shows that $S_{\nwarrow}(S(X))=S\left(S_{\nwarrow}(X)\right)$ for every point set $X$. Furthermore the right outgoing line $L_{i}(X)$ coincides with that of $L_{i-1}(S \ltimes(X))$, for $1 \leq i<\lambda_{0}(X)$.

A proof of Proposition 7 can be given along the lines of Wernisch's argument. For a switch diagram $X$ let $S \nwarrow(X)$ and $S \backslash$ be defined with respect to north-west and south-east shadows. Alternatively these skeletons could be defined by the relations

$$
S_{\nwarrow}(X)=\overline{S(\bar{X})} \text { and } S_{\searrow}(X)=\widehat{S(\widehat{X})} .
$$

If switch diagram $X$ corresponds to a reduced word then $S_{\nwarrow}(S(X))=S(S \ltimes(X))$ and $S \backslash(S(X))=S(S \backslash(X))$. Furthermore the right outgoing line $L_{i}(X)$ coincides with that of $L_{i-1}\left(S_{\nwarrow}(X)\right)$ and the top outgoing line $L_{i}(X)$ coincides with that of $L_{i-1}(S \backslash(X))$, for $1 \leq i<\lambda_{0}(X)$. Unfortunately, the proof of these statements requires a lengthy case analysis.

\section{References}

[1] A. Björner, M. Las Vergnas, B. Sturmfels, N. White and G. M. Ziegler, Oriented Matroids, Encyc. of Math., Vol. 46, Cambridge Univ. Press 1993.

[2] P. Edelman and C. Greene, Balanced tableaux, Adv. in Math., 63 (1987), pp. 42-99.

[3] S. Felsner, The Skeleton of a Reduced Word and a Correspondence of Edelman and Greene, in Formal Power Series and Algebraic Combinatorics, D. Krob et. al ed., Springer 2000, pp. 179-190.

[4] S. Felsner And L. Wernisch, Maximum k-chains in planar point sets: Combinatorial structure and algorithms, SIAM J. on Computing, 28 (1999), pp. 192-209.

[5] S. Fomin And A. N. Kirillov, Reduced words and plane partitions, J. Algebr. Comb., 6 (1997), pp. 311-319.

[6] W. Fulton, Young Tableaux, LMS Student Texts 35, Cambridge Univ. Press, 1997.

[7] J. E. Goodman, Proof of a conjecture of Burr, Grünbaum and Sloane, Discrete Math., 32 (1980), pp. 27-35.

[8] J. E. Goodman and R. Pollack, Semispaces of configurations, cell complexes of arrangements, J. Combin. Theory Ser. A, 37 (1984), pp. 257-293. 
[9] M. Haiman, Dual equivalence with applications, including a conjecture of Proctor, Discrete Math., 99 (1992), pp. 79-113.

[10] D. E. Knuth, Permutations, matrices, and generalized Young tableaux, Pacific J. of Math., 34 (1970), pp. 709-772.

[11] D. E. Knuth, Axioms and Hulls, LNCS, vol. 606, Springer-Verlag, 1992.

[12] W. Kraskiewicz, Reduced decompositions in hyperoctahedral groups, C. R. Acad. Sci., Paris, Ser. I, 309 (1989), pp. 903-907.

[13] A. Lascoux and M. P. Schützenberger, Structure de Hopf de l'anneau de cohomologie et de l'anneau de Grothendieck d'une variete de drapeaux, C. R. Acad. Sci., Paris, Ser. I, 295 (1982), pp. 629-633.

[14] R. Stanley, A combinatorial conjecture concerning the symmetric group, tech. rep., 1982.

[15] R. Stanley, On the number of reduced decompositions of elements of Coxeter groups, Europ. J. Combinatorics, 5 (1984), pp. 359-372.

[16] R. Stanley, Enumerative combinatorics, Vol. 2, Studies in Advanced Mathematics. 62, Cambridge University Press, 1999.

[17] G. Viennot, Une forme géométrique de la correspondance de Robinson-Schensted, in Combinatoire et Représentation du Groupe Symétrique, D. Foata, ed., Lecture Notes in Mathematics 579, 1977, pp. 29-58.

[18] G. Viennot, Chain and antichain families, grids and Young tableaux, in Orders: Description and Roles, North-Holland Math. Stud. 99, Amsterdam-New York, 1984, pp. 409-463.

[19] L. Wernisch, Dominance relation on point sets and aligned rectangles, transparent verlag, Berlin, 1994. ftp://ftp.inf.fu-berlin.de/pub/wernisch. 